\title{
Anatomical variation of the liver with elongated left lobe may be a trap for the ultrasound detection of focal liver lesion
}

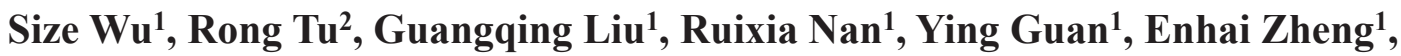 \\ Yanyan Zhao ${ }^{1}$
}

${ }^{1}$ Department of Ultrasound, ${ }^{2}$ Department of Radiology, Affiliated Hospital of Hainan Medical College, Haikou, China

\begin{abstract}
Aims: The aim of this study was to evaluate the impact of anatomical variation of the elongated left lobe of the liver on the ultrasound detection of focal liver lesions. Materials and methods: We evaluated, by ultrasonography (US), 296 patients with elongated left lobe of the liver and 30 patients with focal lesions in the normal length left lobe of the liver. Those patients had undergone prior computed tomography (CT) evaluation. Results: Of the 296 patients evaluated, 34 patients had focal lesions (the size range 11-20 mm) in the distal region of the elongated left lobe of the liver, and none of the lesions was visualized at US examination $(\mathrm{p}<0.001)$. All the focal lesions in the normal length left lobe of the liver of the 30 patients were visualized by US without significant difference between the dimensions established by CT and US ( $\mathrm{p}=0.136)$. Conclusion: Focal lesions in the distal region of elongated left lobe of the liver are difficult to be detected by conventional US examination and the main reason is the impact of anatomical localization on the examination protocol.
\end{abstract}

Keywords: elongated left liver lobe,focal lesion, ultrasound, computed tomography

\section{Introduction}

The liver is the largest solid organ in humans and diseases relating to the liver are numerous. Before the advent of ultrasound (US) imaging, the focal lesions in the liver were difficult to be found and delineated, which lead to a loss of timely management of a considerable number of patients with liver diseases [1-3]. Although computed tomography $(\mathrm{CT})$ and magnetic resonance imaging (MRI) are popular, US is still the most common modality for the initial investigation of the liver worldwide. Normally, the liver can be detected satisfactorily using US except some parts of the subphrenic region $[4,5]$. Previous studies have found that a tumor located in the subphrenic region is not easy to be detected and it may lead to missed diagnosis $[4,5]$. Having realized this, US operators always

Received 03.10.2014 Accepted .13.11.2014

Med Ultrason

2015, Vol. 17, No 1, 12-15

Corresponding author: Size Wu MD

Department of Medical Imaging

Affiliated Hospital of Hainan Medical College,

31, Longhua Road, Haikou 570102, China

Phone: 86-0898-66508833

E-mail: wsz074@aliyun.com scan the subphrenic region of the liver to avoid the missing detection of focal lesions in clinical practice. Occasionally, some focal lesions located at the elongated left lobe of the liver present clearly on images of CT and / or MR, but they cannot be visualized on US. The reason may be associated with the anatomy variation of the liver, but there is no concrete description in literature.

The aim of this study was to evaluate the impact of anatomy variation of the liver on the US detection of focal lesions in the elongated left lobe of the liver.

\section{Materials and methods}

\section{Patient population}

In this retrospective study patients referred consecutively to our hospital during January 2007 and January 2011 in whom the elongated left lobe of the liver was found during CT examination were included. From 319 patients found with elongated left lobe of the liver on CT, 23 patients did not undergo US and were excluded. Finally the study group consisted of 296 patients (181 men, 115 women; mean age, 48 years; range, $18-89$ years). In the control group were included 30 patients (19 men, 11 women; mean age, 43 years; range, 24-81 years) with- 
out elongated left lobe and with small focal lesions (size in11-22 $\mathrm{mm}$ ) detected in the left lobe at CT examination.

\section{Ultrasound examination}

The US examination of the liver and spleen was performed by 4 sonologists with 10-21 years of experience, using Logiq 9 (GE Healthcare, Waukesha, WI), Voluson Expert 730 (GE Healthcare, Piscataway, NJ), Sonos 5500 (Philips Medical systems, Eindhoven, Netherlands), HD11XE (HP Medical systems, Dalian, China), and Mindray DC-8 (Mindray Medical International Limited, Senzheng, China) ultrasound systems, with 2, 3, and $5 \mathrm{MHz}$ convex transducer. The ultrasound operators were blinded regarding CT findings. The liver and spleen of the patients

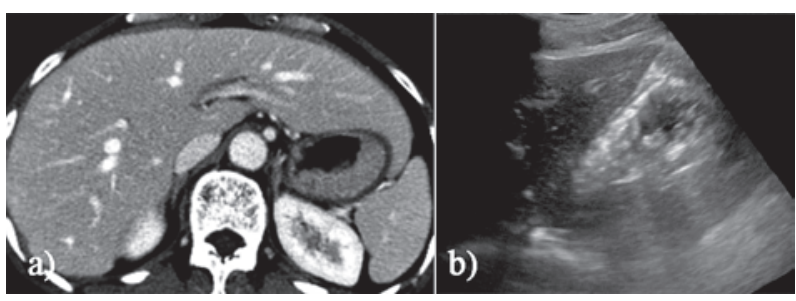

Fig 1. A 34-year-old man with elongated left lobe of the liver: a) $\mathrm{CT}$ shows the elongated left lobe of the liver, exceeding the stomach to the left and approaching the medial side of the spleen; b) the distal region of left lobe of the liver and the spleen are undetectable by ultrasound. were scanned carefully with reference to literature [6]; the operators routinely used inspiration breath hold manoeuvre and all possible windows through the subxiphoid area, subcostal area, and the intercostal space, to bring more of the left lobe under the probe. Representative images were saved digitally in the Picture Archiving and Communication Systems (PACS). The size, echo patterns and associated findings of the liver and splenic lesions were studied. Some of the findings are illustrated in figures 1-3.

\section{CT imaging}

All patients underwent unenhanced CT, and some patients underwent multiphase (unenhanced, and /or arterial phase, portal venous phase, and delayed equilibrium phase) CT for the evaluation of the liver, spleen or other abdominal organs, using a 64-MDCT scanner (LightSpeed VCT, GE Healthcare). Scanning parameters were $64 \times$ $0.625 \mathrm{~mm}$ collimation, $5.0 \mathrm{~mm}$ section thickness and interval, $120 \mathrm{kVp}$, and $300 \mathrm{mAs}$. For the contrast-enhanced portions of the examinations, patients received approximately $80-130 \mathrm{~mL}$ of iohexol $(300 \mathrm{mgI} / \mathrm{mL}$, Yangtze River Pharmaceutical Co., Ltd, Taizhou, China) IV or $100 \mathrm{~mL}$ of iopamidol (370 mgI/mL, Shanghai Bracco sine pharmaceutical limited liability company, Shanghai) by means of a mechanical power injector (Stellant Injector System, Medrad), administered at a rate of 3-4 mL/s, followed by

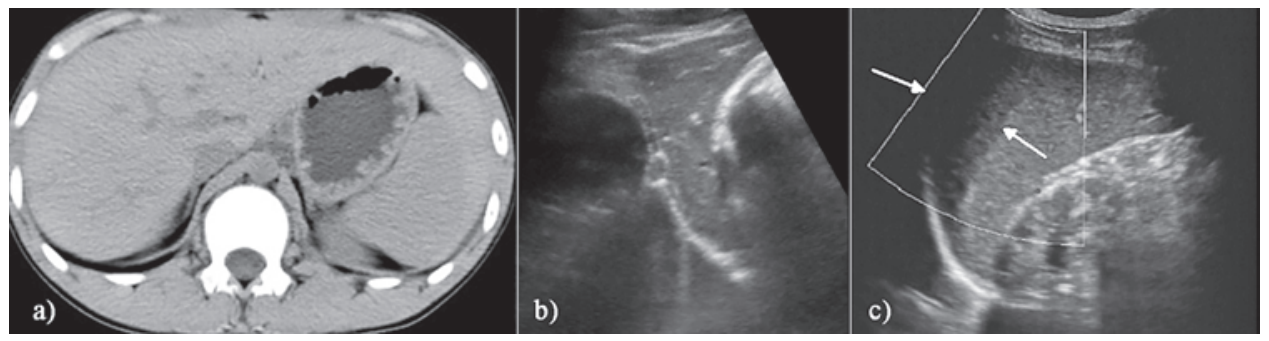

Fig 2. A 25-year-old woman with elongated left lobe of the liver, overlying the spleen. a) CT: the left lobe of liver elongates exceeding the stomach to the left and draping the spleen; b) ultrasound examination: the spleen and the liver overlying the spleen cannot be visualized when scanning from subxiphoid area; c) the spleen and the liver overlying the spleen can be only partially visualized when scanning from the left flank through the spleen and the intercostal area (arrows).

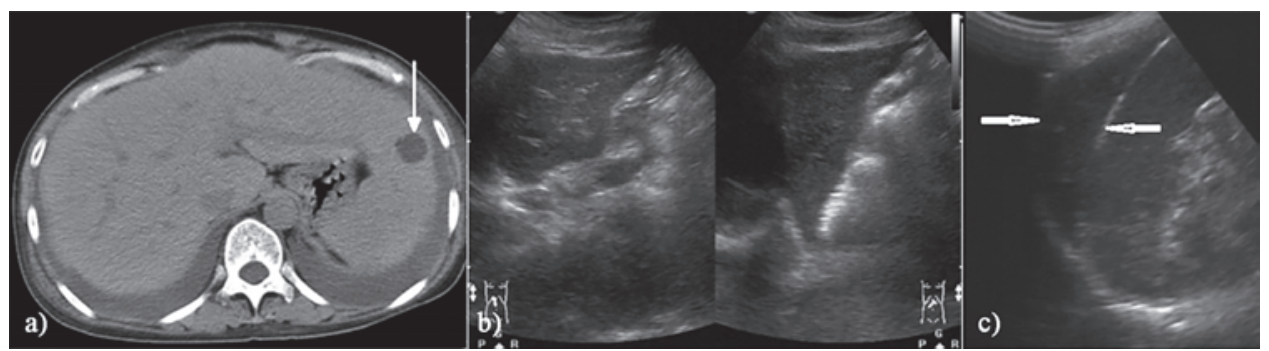

Fig 3. A 31-year-old woman with liver cystic lesion. a) CT: left lobe of the liver with a round hypodense cystic lesion (arrow) elongates exceeding the stomach and draping the spleen; b) ultrasonography: the spleen and the liver overlying the spleen cannot be visualized from subxiphoid (right plot and left plot), and the cystic lesion is undetectable; c) left lobe of the liver presenting isoechogenicity is well visualized, but the cystic lesion is invisible. 
$15-20 \mathrm{~mL}$ of saline flush. All helical studies were started at the top of the liver and proceeded in a cephalocaudal direction; unenhanced and three-phase contrast-enhanced helical scans of the entire liver were obtained.

\section{Images study}

Taking findings on CT images as reference, findings in the left lobe of the liver (elongated or of normal length) on images of CT and US were compared, and concordance was analyzed.

An elongated left lobe of the liver is defined when the left lobe of the liver exceeds the stomach to the left and / or reaches the superior pole of the spleen on image of CT.

\section{Statistical analysis}

The continuous variables were expressed as the mean values \pm standard deviations. The size of the largest lesion in the elongated left lobe of the liver and the normal length left lobe of the liver was compared using independent samples $t$ test, and the US detected focal lesions between the elongated left lobe of the liver and normal length left lobe of the liver were compared using the Chi-square test. A $P$ level of 0.05 or less (two-sided) was considered statistically significant. All analyses were performed with SPSS software (version 20; IBM corporation, Armonk, New York, USA).

The retrospective study was approved by our institutional review board and informed consent of the patients was waived.

\section{Results}

Parameters of the lesions and the comparison between $\mathrm{CT}$ and US in detection of the focal liver are listed in Table I. In the 296 patients evaluated, 34 patients had one or several lesions (the size range $11-20 \mathrm{~mm}$ ) in the distal part of the elongated left lobe of the liver at CT examination. None of these lesions was visualized at US examination $(p<0.001)$. Focal lesions in the normal length left lobe of the liver of the control group (30 patients) were all visualized by US $(\mathrm{p}=1.000)$. No significant differences were obtained in the measurement of lesions using the two imaging techniques $(\mathrm{p}=0.136)$.

\section{Discussions}

Generally the liver locates dominantly at the right upper quadrant of the abdomen, and it seldom extends to the lodge of the spleen $[7,8]$. Nevertheless, according to our experience in the study of CT images of the liver and spleen, the liver with an elongated left lobe is not a rare situation. In some patients, the left lobe of liver extends towards left, over the stomach, and even drapes on the spleen $[6,9]$. Due to the presence of the air in stomach and colon, the spleen is undetectable or obscured from subxiphoid and left subcostal area at US scan, and so is the elongated left lobe of the liver. This is the reason why a lesion in the distal region of an elongated left lobe of the liver is easily overlooked at US examination. The results of this study suggest that the lesions in the distal region of elongated left lobe of the liver may not be visualized at US scanning. Meanwhile, all focal lesions in the normal length left lobe of the liver were visible on US.

The presence of the elongated left lobe of the liver not only can be misinterpreted as subcapsular splenic hematoma, etc. [7-10], but also can cause missed diagnosis of focal lesions at US examination. Recently we proposed a method to improve the visualization of the distal part of elongated left lobe of the liver through intake of sufficient water for distending the stomach, a method that can improve the detection of the focal lesion [11]. In clinical practice, if the operator realizes the existence of an elongated left lobe of the liver and the likeliness of a misdiagnosis of focal liver lesion, the stomach must be filled with sufficient water.

We believe that the causes why lesions in the elongated left lobe of the liver are not visualized at US, are due to the anatomical variation of the liver: 1) the presence of gas impede US transmission and imaging- the elongated left lobe of the liver locates at high position and medial to the spleen, and its distal region is hardly to be visualized completely in the presence of the stomach, colon, and lung (organs that contain a great amount of air); 2) the presence of the ribs impede the US beam transmission and affect US imaging; 3 ) the lesions situated in the remote field of the $n$ elongated left lobe of the liver are scanned with US from the left subcostal area and the resolution in the remote field is low; 4) probably the frequency of the isoechoic focal liver lesions is higher in the elongated left lobe of the liver which makes its not visible.

The potential limitations of this study are the relatively small sample and absence of larger lesions, which may cause sample selection bias. Therefore, additional large prospective studies are required to fully determine the in-

Table I. Comparison of ultrasound detected focal lesions between the elongated left lobe of the liver and the normal length left lobe of the liver.

\begin{tabular}{llll}
\hline Location & Size $(\mathbf{m m})$ & Visualization on CT $(\mathbf{n})$ & Visualization on US (n) \\
\hline Lesion in elongated left lobe & $16.09 \pm 2.25$ & 34 & 0 \\
Lesion in normal length left lobe & $16.32 \pm 2.54$ & 30 & 30 \\
\hline
\end{tabular}

$\mathrm{n}$ - number of the focal lesions 
fluence of the anatomical variation of elongated left lobe of the liver on the US detection of focal lesions in the liver.

In conclusion, focal lesions in the elongated left lobe of the liver are difficult to be detected at US, the main reason being the presence of air from the bowels and lungs in the region. The elongated left lobe of the liver locates around the organs filled with gas, which compromises the scanning acoustic window and this may lead to failure in the detection of focal liver lesions. We speculated that if tumor harboring is suspected in a patient with anatomical variation of the elongated left lobe of the liver, the US examiner could improve visualization of the elongated left lobe of the liver by distending the stomach with sufficient water. An examiner of abdominal US should be aware of the anatomical variation of the elongated left lobe of the liver and be vigilant for missed diagnosis.

\section{Conflict of interest: none}

Acknowledgements: The authors would like to express their gratitude to ultrasound radiologists Li Huang, Ying Wan, Shibin Lin, Chuan Fu, and Kailiang Chen for their assistance.

\section{References}

1. Koslin DB, Berland LL. Duplex Doppler examination of the liver and portal venous system. J Clin Ultrasound 1987; 15: 675-686.
2. Shin DS, Jeffrey RB, Desser TS. Pearls and pitfalls in hepatic ultrasonography. Ultrasound Q 2010; 26: 17-25.

3. Bartolotta TV, Taibbi A, Midiri M, Matranga D, Solbiati L, Lagalla R. Indeterminate focal liver lesions incidentally discovered at gray-scale US: role of contrast-enhanced sonography. Invest Radiol 2011; 46: 106-115.

4. Lee MW, Kim YJ, Park HS, et al. Targeted sonography for small hepatocellular carcinoma discovered by CT or MRI: factors affecting sonographic detection. AJR Am J Roentgenol 2010; 194: W396-W400.

5. Kang TW, Rhim H, Lee MW, Kim W, Park JG. Predicting coverage of transverse subcostal sonography with the use of previous computed tomography before a sonographic liver examination: a prospective study. J Ultrasound Med 2013; 32: 2053-2061.

6. Dănilă M. The ultrasound examination of the spleen. Med Ultrason 2010; 12: 253-254.

7. Chaudhuri TK, Bobbitt JV. Marked enlargement of the left lobe of liver causing a false-positive spleen image. Radiology 1976; 119: 169-170.

8. Jacobs S, Kirsh J, Goldfinger M, Rosen I. Pitfall in the investigation of splenic trauma. J Can Assoc Radiol 1984; 35 : 378-379.

9. Roberts JL, Dalen K, Bosanko CM, Jafir SZ. CT in abdominal and pelvic trauma. Radiographics 1993; 13: 735-752.

10. Hammond LJ. III. Congenital elongation of the left lobe of the liver. Ann Surg 1905; 41: 31-35.

11. Wu S, Tu R, Zheng E, Liu G. Approach of improving sonographic visualization of elongated left lobe of the liver and its implications. Journal of Medical Ultrasonics 2013; 40: 425-428. 